\title{
Droplet spreading under weak slippage: the optimal asymptotic propagation rate in the multi-dimensional case
}

\author{
GÜNTHER GRÜN ${ }^{\dagger}$ \\ Universität Bonn, Institut für Angewandte Mathematik, Beringstr. 6, 53115 Bonn, Germany
}

[Received 1 August 2001]

\begin{abstract}
We prove optimal estimates on the growth rate of the support of solutions to the thin-film equation $u_{t}+\operatorname{div}\left(|u|^{n} \nabla \Delta u\right)=0$ in space dimensions $N=2$ and $N=3$ for parameters $n \in[2,3)$ which correspond to Navier's slip condition $(n=2)$ or certain variants modeling weaker slippage effects. Our approach relies on a new class of weighted energy estimates. It is inspired by the onedimensional technique of Hulshof and Shishkov Adv. Diff. Equations 3, (1998) 625-642, and it simplifies their method, mainly with respect to basic integral estimates to be used.
\end{abstract}

Keywords: fourth-order degenerate parabolic equations; finite speed of propagation; thin films

\section{Introduction and statement of the main result}

In this paper we study the asymptotic behavior for $t \rightarrow \infty$ of the support of solutions to the Cauchy problem

$$
u_{t}+\operatorname{div}\left(|u|^{n} \nabla \Delta u\right)=0 \quad \text { in } \mathbb{R}^{N} \times \mathbb{R}^{+}
$$

with compactly supported initial data $u_{0} \in H^{1}\left(\mathbb{R}^{N} ; \mathbb{R}_{0}^{+}\right)$in space dimensions $N=2$ and $N=3$. Equation (1.1) is a model problem for a class of fourth-order degenerate parabolic equations arising in materials sciences and fluid dynamics (see [10, 13,22] and references therein). In the formulation above, it describes the surface tension driven evolution of the height $u$ of a thin film of viscous liquid spreading on a horizontal surface. In general, the exponent $n$ is assumed to be a positive number. From the physical point of view, the cases $n=2$ and $n=3$ are distinguished. Taken with a grain of salt, the former one corresponds to the assumption of Navier's slip condition, the latter one to the assumption of a no-slip condition at the liquid solid interface. As the total mass is conserved under that evolution and since the equation is invariant under the scalings

$$
x=K \hat{x}, \quad t=K^{\gamma} \hat{t}, \quad u=K^{-N} \hat{u}
$$

with $\gamma=4+n N$, the existence of a compactly supported self-similar solution of the form

$$
u(x, t)=t^{-\frac{1}{4+n N}} U\left(\frac{x}{t^{\frac{1}{4+n N}}}\right)
$$

would be expected for all values of $n>0$. Surprisingly, this self-similar solution only exists for $n$ in the interval $(0,3)$ as was proven by Bernis et al. [7] in space dimension $N=1$ and by Ferreira

\footnotetext{
Email: gg@math.uni-bonn.de
} 
and Bernis [15] in the multi-dimensional case. In contrast, for $n \geqslant 3$ solutions are expected to have a support constant in time. As the exponent $n=3$ corresponds to the assumption of a noslip condition, this result is consistent with the observation made by Dussan and Davis [14] that a no-slip condition causes infinite energy dissipation for moving droplets. However, this is not the only phenomenon indicating that the solution's behavior is sensitive to the exponent $n$. Indeed, the analytical tools used to prove results on existence, non-negativity or on the qualitative behavior of solutions depend on the fact whether $n \in(0,2)$ or $n \in[2,3)$. In the former range, finite speed of propagation, optimal growth rates for the solution's support or occurrence of a waiting time phenomenon could be proven in all the physically relevant space dimensions (see $[3,6]$ for results in one space dimension, $[9,11]$ for results in higher space dimensions). To put it concisely, these results were based on certain variants of a particular integral estimate which is sometimes called the entropy estimate (see $[2,8,10]$ for further details on that estimate). In the range $n \in[2,3$ ), however, this entropy estimate cannot be used any more and therefore it seems that the analytical arguments have to be based on the only remaining integral estimate which is the energy estimate and which reads as follows:

$$
\frac{1}{2} \int_{\mathbb{R}^{N}}|\nabla u(\cdot, T)|^{2}+\int_{0}^{T} \int_{\mathbb{R}^{N}} u^{n}|\nabla \Delta u|^{2} \leqslant \frac{1}{2} \int_{\mathbb{R}^{N}}\left|\nabla u_{0}\right|^{2} .
$$

By virtue of Bernis' interpolation inequalities (see [5])

$$
\begin{aligned}
& \int_{\Omega} v^{n-4} v_{x}^{6} \leqslant C \int_{\Omega} v^{n-1}\left|v_{x x}\right|^{3}, \\
& \int_{\Omega} v^{n-1}\left|v_{x x}\right|^{3} \leqslant C \int_{\Omega} v^{n} v_{x x x}^{2},
\end{aligned}
$$

the second term on the left-hand side of (1.4) becomes utilizable for Gagliardo-Nirenberg-type arguments, and Bernis [4] and Hulshof and Shishkov [20] succeeded in proving in one space dimension a qualitative result on finite speed of propagation and an optimal estimate on the growth of the solutions's support, respectively (see also Andreucci and Tedeev [1] for the latter result using a different approach).

Just recently, the author of this note was able to establish a multi-dimensional equivalent of the interpolation inequalities (1.5) and (1.6) (see [18]). This was the key ingredient to prove in [19] the following existence result for the Cauchy problem in the multi-dimensional case.

THEOREM 1.1 Let $n \in\left(2-\sqrt{1-\frac{N}{8+N}}, 3\right), N<4$, and assume $u_{0} \in H^{1}\left(\mathbb{R}^{N}\right)$ to be non-negative with compact support in the sense that $u_{0}(x)=0$ almost everywhere on $\mathbb{R}^{N} \backslash B_{R_{0}}(0)$ for a positive number $R_{0}$. Then, a non-negative function $u$ exists that has the following properties: 
(i) Regularity:

$$
\begin{aligned}
& u_{t} \in L^{2}\left(\mathbb{R}^{+} ;\left(W^{1, p}(\Omega)\right)^{\prime}\right) \text { for } p>\frac{4 N}{2 N+n(2-N)} \text { and any } \Omega \subset \subset \mathbb{R}^{N}, \\
& u \in L^{\infty}\left(\mathbb{R}^{+} ; H^{1}\left(\mathbb{R}^{N}\right)\right), \\
& \nabla \Delta u^{\frac{n+2}{2}} \in L^{2}\left(\mathbb{R}^{N} \times \mathbb{R}^{+}\right), \\
& \nabla u^{\frac{n+2}{6}} \in L^{6}\left(\mathbb{R}^{N} \times \mathbb{R}^{+}\right), \\
& D^{2} u^{\frac{\alpha+n+1}{2}} \in L^{2}\left(\mathbb{R}^{N} \times \mathbb{R}^{+}\right) \text {for any } \alpha \in(\max \{-1,1 / 2-n\}, 2-n), \\
& \nabla u^{\frac{\alpha+n+1}{4}} \in L^{4}\left(\mathbb{R}^{N} \times \mathbb{R}^{+}\right) \text {for any } \alpha \in(\max \{-1,1 / 2-n\}, 2-n), \\
& J=\left\{\begin{array}{ll}
u^{n} \nabla \Delta u & \text { on }[u>0] \\
0 & \text { on }[u=0]
\end{array} \quad \in L^{2}\left(\mathbb{R}^{+} ; L^{q}\left(\mathbb{R}^{N}\right)\right)\right. \\
& \text { for any } 1<q<\frac{4 N}{2 N+n(N-2)} .
\end{aligned}
$$

(ii) $u$ is a solution to the Cauchy problem in the sense that

$$
\int_{0}^{T}\left\langle u_{t}, \phi\right\rangle_{\left(W^{1, p}(B(0))\right)^{\prime} \times W^{1, p}(B(0))}-\int_{[u>0]_{T}} u^{n} \nabla \Delta u \nabla \phi=0
$$

for $p>\frac{4 N}{2 N+n(2-N)}$, arbitrary $T>0$ and for all test functions $\phi$ contained in $L^{2}\left((0, T) ; W^{1, \infty}\left(\mathbb{R}^{N}\right)\right)$ such that $\bigcup_{t \in(0, T)} \operatorname{supp} \phi(., t) \subset B(0)$, where $B(0)$ is an arbitrary ball centered in the origin $0 \in \mathbb{R}^{N}$.

(iii) The solution $u$ attains initial data $u_{0}$ in the sense that

$$
\lim _{t \searrow 0} u(\cdot, t)=u_{0}(\cdot) \quad \text { in } L_{\mathrm{loc}}^{\beta}\left(\mathbb{R}^{N}\right)
$$

for arbitrary $1 \leqslant \beta<\frac{2 N}{N-2}$.

Moreover, the solutions constructed in Theorem 1.1 have the property of finite speed of propagation in the following weak sense (see [19] and the forthcoming paper [17]).

DEFinition 1.2 Let $v: \mathbb{R}^{N} \times(0, \infty) \rightarrow \mathbb{R}$ be a non-negative function and assume that for every $t \in[0, \infty)$ the function $v(., t)$ has compact support. We say that $v$ has finite speed of propagation iff for each ball $\overline{B\left(x_{0}, R_{0}\right)}, x_{0} \in \mathbb{R}^{N}, R_{0}>0$, that contains supp $v(\cdot, 0)$, a continuous, monotonically increasing function $R:[0, \infty) \rightarrow \mathbb{R}_{0}^{+}, R(0)=0$, exists such that

$$
\operatorname{supp} v(., t) \subset \overline{B\left(x_{0}, R_{0}+R(t)\right)} \text {. }
$$

It is the purpose of this paper to establish a quantitative estimate on the maximum growth rate of the solution's support. More precisely, the following result will be obtained.

THEOREM 1.3 Let $n \in\left(2-\sqrt{1-\frac{N}{8+N}}, 3\right), N<4$ and assume $u$ to be a solution of the Cauchy problem (1.1) in the sense of Theorem 1.1. Moreover, suppose that initial data $u_{0}$ satisfy

$$
\operatorname{supp} u_{0} \subset B\left(0, R_{0}\right)
$$


for a positive number $R_{0}$.

Then a positive constant $C$ exists which only depends on $n, N$ and the mass of initial data such that

$$
\operatorname{supp} u(\cdot, t) \subset B\left(0, R_{0}+C \cdot t^{\frac{1}{4+n N}}\right)
$$

for all $t>0$.

Remarks.

(1) Note that the exponent $\alpha=\frac{1}{4+n N}$ is consistent with the scaling in (1.2) and therefore optimal.

(2) Analogous results for $0<n<2$ and $N \in\{2,3\}$ were established in Bertsch et al. [9].

(3) For $n \geqslant 3$ it is conjectured that the solution's support is constant in time.

(4) Physically, the assumption of a slip condition

$$
\left.\vec{v}_{\text {hor }}\right|_{z=0}=\left.\beta h^{n-2} \cdot \frac{\partial \vec{v}_{\text {hor }}}{\partial z}\right|_{z=0}
$$

entails ${ }^{\dagger}$ the thin-film equation

$$
h_{t}+\operatorname{div}\left(\left(h^{3}+\beta h^{n}\right) \nabla \Delta h\right)=0 .
$$

This equation obviously loses the scaling properties (1.2). Hence, self-similar solutions cease to exist. In the case of (1.18), Theorem 1.3 can therefore serve to describe the propagation rate for larger values of $t$ when the exponent $n$ becomes dominant. For an intermediate time-scale, Giacomelli and Otto [16] recently proved in one space dimension that the apparent support, i.e. the set $\{x \in \mathbb{R}: h(\cdot, t)>\beta\}$, increases with the rate $\left(\frac{t}{\log 1 / t}\right)^{1 / 7}$.

(5) The validity restriction for values of $(N, n)$ is inherited from identical conditions in the existence result Theorem 1.1.

Our approach simplifies and extends the method of Hulshof and Shishkov [20] who proved a similar result in one space dimension. For instance, we will not need to prove $L^{2}\left(\mathbb{R}^{N} \times(0, T)\right)$ estimates for the quantity $\nabla \Delta\left(\eta \cdot u^{\frac{n+2}{2}}\right)$ where $\eta$ is an appropriate cut-off function, which-in the multi-dimensional case - would be a rather tedious task. Nor will we rely on estimates of third-order derivatives of certain powers of the solution. In this way, further technical difficulties can be avoided which would naturally arise in the multi-dimensional setting as soon as interpolation arguments are to be applied. These are due to the fact that only $\nabla \Delta u^{\frac{n+2}{2}}$ but not the entire tensor of thirdorder derivatives can be controlled. Instead, we will base our argument on the $L^{6}\left(\mathbb{R}^{N} \times(0, T)\right)$ integrability of $\nabla u^{\frac{n+2}{6}}$ which could be established in [19].

On account of these changes in strategy, we will present the proof in some detail. In Section 2, we recall the weighted energy estimate from [19] and derive-in combination with an appropriate scaled version of Poincaré's inequality on annuli-the integral estimate essential for the sequel. By means of an iteration lemma due to Stampacchia (see [21]), we prove in Section 3 a preliminary estimate on the radius of the solution's support. As this result still depends on certain integral

\footnotetext{
"Here, $\vec{v}_{\text {hor }}$ denotes the horizontal velocity component of the fluid flow, $\beta$ is a positive parameter and $z$ stands for the vertical coordinate. For $n=2$, the classical slip condition of Navier is recovered.
} 
quantities involving the solution $u$, we first have to estimate these quantities only in terms of initial mass and time before Theorem 1.3 can be proven. Both will be accomplished in Section 4. Finally, the Appendix contains a number of auxiliary results frequently used within the text.

Throughout the paper, we will use the standard notation for Sobolev spaces. $\vec{v}$ stands for the unit outer normal vector to a domain $\Omega$, and we write $B(\varrho)$ for the ball with radius $\varrho$ centered in the origin. $A\left(\varrho_{0}, \varrho_{1}\right)$ denotes for $\varrho_{1}>\varrho_{0}>0$ the annulus $B\left(\varrho_{1}\right) \backslash \overline{B\left(\varrho_{0}\right)}$. Sometimes, we abbreviate $\frac{\partial}{\partial x} u$ by $u_{x}$, and we also write $L^{1}$ instead of $L^{1}\left(\mathbb{R}^{N}\right)$ when no misunderstanding can occur. Finally, $I_{T}(\varrho)$ stands for $\int_{0}^{T} \int_{\mathbb{R}^{N} \backslash B(\varrho)} u^{n+2} \mathrm{~d} x \mathrm{~d} t$.

\section{The weighted energy estimate and some of its variants}

In this section, we will provide the key result to prove a recursive estimate of the form

$$
I_{T}(\varrho+\delta) \leqslant \frac{C\left(I_{T}(\varrho)-I_{T}(\varrho+\delta)\right)^{1+\beta} \cdot F(T)}{\delta^{m}}
$$

with positive quantities $m, \beta, F(T)$, which is—as in [20]—fundamental for a future application of Stampacchia's iteration lemma.

To this end, let us consider for positive $\varrho, \delta \in \mathbb{R}$ a smooth localization function $\psi_{\varrho \delta}$ having the following properties:

$$
\begin{aligned}
& \psi_{\varrho \delta} \equiv 0 \text { on } B(\varrho), \\
& 0 \leqslant \psi_{\varrho \delta} \leqslant 1 \text { on } A(\varrho, \varrho+\delta), \\
& \psi_{\varrho \delta} \equiv 1 \text { on } \mathbb{R}^{N} \backslash B(\varrho+\delta), \\
&\left\|\nabla \psi_{\varrho \delta}\right\|_{\infty} \leqslant C \delta^{-1} \\
&\left\|D^{2} \psi_{\varrho \delta}\right\|_{\infty} \leqslant C \delta^{-2}
\end{aligned}
$$

In [19], the following integral estimate for solutions to the auxiliary problem

$$
\begin{array}{cc}
w_{t}+\operatorname{div}\left(|w|^{n} \nabla \Delta w\right)=0 & \text { in } B(k) \times \mathbb{R}^{+} \\
\frac{\partial}{\partial \nu} w=\frac{\partial}{\partial v} \Delta w=0 & \text { on } \partial B(k) \times \mathbb{R}^{+} \\
w_{0} \in H^{1}\left(B(k) ; \mathbb{R}_{0}^{+}\right)
\end{array}
$$

and $k>\varrho+\delta$ has been proved:

$$
\begin{aligned}
\int_{B(k)} \psi_{\varrho \delta}^{6}|\nabla w(\cdot, T)|^{2}+ & C^{-1}\left\{\int_{0}^{T} \int_{B(k)} \psi_{\varrho \delta}^{6}\left|\nabla w^{\frac{n+2}{6}}\right|^{6}+\int_{0}^{T} \int_{B(k)} \psi_{\varrho \delta}^{6}\left|\nabla \Delta w^{\frac{n+2}{2}}\right|^{2}\right\} \\
\leqslant & \int_{B(k)} \psi_{\varrho \delta}^{6}\left|\nabla w_{0}\right|^{2} \\
& +C \int_{0}^{T} \int_{B(k)} w^{n+2}\left\{\left|\nabla \psi_{\varrho \delta}\right|^{6}+\left|D^{2} \psi_{\varrho \delta}\right|^{2}\left|\nabla \psi_{\varrho \delta}\right|^{2} \psi_{\varrho \delta}^{2}+\left|D^{2} \psi_{\varrho \delta}\right|^{3} \psi_{\varrho \delta}^{3}\right\} .
\end{aligned}
$$

Note that the property $\frac{\partial}{\partial \nu} \psi_{\varrho \delta}=0$ on $\partial B(k)$ is essential for the validity of (2.4). 
Following the lines of the existence proof for solutions to the Cauchy problem in [19], it becomes evident that the solution $u$ constructed in Theorem 1.1 satisfies the following estimate for arbitrary $\varrho, \delta>0$ and for arbitrary positive times $T$ :

$$
\begin{aligned}
\int_{\mathbb{R}^{N}} \psi_{\varrho \delta}^{6}|\nabla u(\cdot, T)|^{2} & +C^{-1}\left\{\int_{0}^{T} \int_{\mathbb{R}^{N}} \psi_{\varrho \delta}^{6}\left|\nabla u^{\frac{n+2}{6}}\right|^{6}+\int_{0}^{T} \int_{\mathbb{R}^{N}} \psi_{\varrho \delta}^{6}\left|\nabla \Delta u^{\frac{n+2}{2}}\right|^{2}\right\} \\
\leqslant & \int_{\mathbb{R}^{N}} \psi_{\varrho \delta}^{6}\left|\nabla u_{0}\right|^{2} \\
& +C \int_{0}^{T} \int_{\mathbb{R}^{N}} u^{n+2}\left\{\left|\nabla \psi_{\varrho \delta}\right|^{6}+\left|D^{2} \psi_{\varrho \delta}\right|^{2}\left|\nabla \psi_{\varrho \delta}\right|^{2} \psi_{\varrho \delta}^{2}+\left|D^{2} \psi_{\varrho \delta}\right|^{3} \psi_{\varrho \delta}^{3}\right\} .
\end{aligned}
$$

From (2.2) and (2.5), we immediately infer the following lemma.

LEMMA 2.1 Let $\varrho>0, \delta>0$ and $T>0$ and assume $u$ to be a solution of (1.1) in the sense of Theorem 1.1 to initial data satisfying (1.16). Then positive constants $d=d(n, N)$ and $C=C(n, N)$ exist such that

$$
\begin{gathered}
\sup _{t \in(0, T)} \int_{\mathbb{R}^{N} \backslash B(\varrho+\delta)}|\nabla u(\cdot, t)|^{2} \\
+d(n, N) \int_{0}^{T} \int_{\mathbb{R}^{N} \backslash B(\varrho+\delta)}\left|\nabla u^{\frac{n+2}{6}}\right|^{6} \\
\leqslant \frac{C(n, N)}{\delta^{6}} \int_{0}^{T} \int_{A(\varrho, \varrho+\delta)} u^{n+2} .
\end{gathered}
$$

By virtue of the qualitative result on finite speed of propagation presented in [19], there exists a continuous monotone function $R:(0, \infty) \rightarrow\left[R_{0}, \infty\right)$ such that

$$
\operatorname{supp} u(\cdot, t) \subset B(0, R(t)) \text {. }
$$

An application of Lemma A.1 of the Appendix entails the following estimate.

LEMMA 2.2 Let $u$ be a solution of (1.1) in the sense of Theorem 1.1 to initial data satisfying (1.16). Let $\tilde{K}>1$ be a positive parameter and assume that $\varrho$ and $\delta$ are positive constants with the properties $\varrho>R_{0}$ and $\varrho+\delta>\frac{R(T)}{\tilde{K}}$ for arbitrary, but fixed $T>0$, where $R(T)$ is the quantity introduced in (2.7). Then, positive constants $d=d(n, N, \tilde{K})$ and $C=C(n, N, \tilde{K})$ exist such that

$$
\begin{gathered}
\sup _{t \in(0, T)} \int_{\mathbb{R}^{N} \backslash B(\varrho+\delta)} u(\cdot, t)^{2}+d \cdot(R(T)-\varrho-\delta)_{+}^{2} \int_{0}^{T} \int_{\mathbb{R}^{N} \backslash B(\varrho+\delta)}\left|\nabla u^{\frac{n+2}{6}}\right|^{6} \\
\leqslant \frac{C \cdot(R(T)-\varrho-\delta)_{+}^{2}}{\delta^{6}} \int_{0}^{T} \int_{A(\varrho, \varrho+\delta)} u^{n+2} .
\end{gathered}
$$

\section{Stampacchia's iteration lemma and a first estimate on the support}

Combining Lemma 2.2 with an appropriate version of Gagliardo-Nirenberg's inequality, we may establish the desired estimate in the spirit of (2.1). 
LEMMA 3.1 Let $u$ be a solution of (1.1) in the sense of Theorem 1.1 to initial data satisfying (1.16) and assume $T>0$ to be arbitrary, but fixed. Suppose that $\varrho>R_{0}, \delta>0$, and $\varrho+\delta>\frac{R(T)}{\tilde{K}}$ with a positive parameter $\tilde{K}>1$. Then a positive constant $C_{1}=C_{1}(n, N, \tilde{K})$ exists such that

$$
I_{T}(\varrho+\delta) \leqslant C_{1} \cdot T^{\frac{12}{n N+12}}(R(T)-\varrho-\delta)_{+}^{\frac{12(n+2)}{n N+12}}\left(\frac{I_{T}(\varrho)-I_{T}(\varrho+\delta)}{\delta^{6}}\right)^{\frac{(6+N) n+12}{n N+12}} .
$$

Proof. Let us first estimate the left-hand side of (3.1) in terms of the quantities appearing on the left-hand side of (2.8). We apply Gagliardo-Nirenberg's inequality in the form presented in Theorem 7.4.1 of [19]. Introducing $w:=u^{\frac{n+2}{6}}$, we obtain

$$
\left(\int_{\mathbb{R}^{N} \backslash B(\varrho+\delta)} w^{6}\right)^{1 / 6} \leqslant C\left(\int_{\mathbb{R}^{N} \backslash B(\varrho+\delta)} w^{\frac{12}{n+2}}\right)^{\frac{n+2}{12}(1-\theta)}\left(\int_{\mathbb{R}^{N} \backslash B(\varrho+\delta)}|\nabla w|^{6}\right)^{\frac{\theta}{6}},
$$

where

$$
\theta=\frac{n N}{n N+12}
$$

Rewriting this estimate in terms of $u$ and integrating with respect to time gives

$$
\begin{gathered}
\int_{0}^{T} \int_{\mathbb{R}^{N} \backslash B(\varrho+\delta)} u^{n+2} \leqslant C\left(\int_{0}^{T} \int_{\mathbb{R}^{N} \backslash B(\varrho+\delta)}\left|\nabla u^{\frac{n+2}{6}}\right|^{6}\right)^{\frac{n N}{n N+12}}\left(\int_{0}^{T}\left(\int_{\mathbb{R}^{N} \backslash B(\varrho+\delta)} u^{2}\right)^{\frac{n+2}{2}}\right)^{\frac{12}{n N+12}} \\
\leqslant C \cdot T^{\frac{12}{n N+12}}\left(\int_{0}^{T} \int_{\mathbb{R}^{N} \backslash B(\varrho+\delta)}\left|\nabla u^{\frac{n+2}{6}}\right|^{6}\right)^{\frac{n N}{n N+12}}\left(\sup _{t \in(0, T)} \int_{\mathbb{R}^{N} \backslash B(\varrho+\delta)} u^{2}\right)^{\frac{6(n+2)}{n N+12}}
\end{gathered}
$$

Combining (3.3) with (2.8), it follows that

$$
\begin{aligned}
I_{T}(\varrho+\delta) \leqslant C_{0} & \cdot T^{\frac{12}{n N+12}}\left(\frac{C(n, N, \tilde{K})}{\delta^{6}}\left(I_{T}(\varrho)-I_{T}(\varrho+\delta)\right)\right)^{\frac{n N}{n N+12}} \cdot(R(T)-\varrho-\delta)_{+}^{\frac{12(n+2)}{n N+12}} \\
& *\left(\frac{C(n, N, \tilde{K})}{\delta^{6}}\left(I_{T}(\varrho)-I_{T}(\varrho+\delta)\right)\right)^{\frac{6(n+2)}{n N+12}} \\
= & C_{0} T^{\frac{12}{n N+12}}(R(T)-\varrho-\delta)_{+}^{\frac{12(n+2)}{n N+12}}\left(\frac{C(n, N, \tilde{K})}{\delta^{6}}\left(I_{T}(\varrho)-I_{T}(\varrho+\delta)\right)\right)^{\frac{(6+N) n+12}{n N+12}}
\end{aligned} .
$$

This proves the claim.

Let us now prove a first estimate on $R(T)$. We need the following version of Stampacchia's iteration lemma (see [20,21]).

LEMMA 3.2 Suppose that the non-negative and non-increasing function $J$ satisfies with fixed real numbers $r_{0}$ and $0<\theta<1$ the estimate

$$
J(r+J(r))<\theta J(r) \quad \text { for all } r>r_{0} .
$$


Then, for any $r_{1} \geqslant r_{0}$,

$$
J(r)=0 \quad \text { for all } r>r_{1}+\frac{J\left(r_{1}\right)}{1-\theta} .
$$

Our result reads as follows.

LEMMA 3.3 Let $\tilde{K}>1$ be a fixed parameter and assume $u$ to be a solution of (1.1) in the sense of Theorem 1.1 to initial data satisfying (1.16). Then a positive constant $C=C(n, N, \tilde{K})$ exists such that

$$
R(T) \leqslant \varrho_{1}+C(n, N, \tilde{K}) T^{\frac{2}{(4+N) n+8}} \cdot I_{T}\left(\varrho_{1}\right)^{\frac{n}{(4+N) n+8}}
$$

for all $\varrho_{1}>\max \left\{R_{0}, \frac{R(T)}{\tilde{K}}\right\}$.

Remark. By construction, we have for arbitrary $\varrho \geqslant \varrho_{1}$ and $\delta>0$ :

$$
\varrho+\delta>\frac{R(T)}{\tilde{K}} \text { and }(R(T)-\varrho-\delta)_{+} \leqslant\left(R(T)-\varrho_{1}\right)_{+} .
$$

Proof. The proof is inspired by the corresponding result in [20]. Without loss of generality we may assume that $R(T)>\varrho_{1}$. Consider for given $\varrho_{1}>\max \left\{R_{0}, \frac{R(T)}{\tilde{K}}\right\}$ the function

$$
J_{T}(\varrho):=F(T)^{1 / m} \cdot I_{T}(\varrho)^{\beta / m}=\left(R(T)-\varrho_{1}\right)_{+}^{\frac{2(n+2)}{(6+N) n+12}} \cdot T^{\frac{2}{(6+N) n+12}} \cdot I_{T}(\varrho)^{\frac{n}{(6+N) n+12}},
$$

where

$$
F(T):=\left(R(T)-\varrho_{1}\right)^{\frac{12(n+2)}{n N+12}} \cdot T^{\frac{12}{n N+12}}, \quad m:=\frac{6((6+N) n+12)}{n N+12}, \beta:=\frac{6 n}{n N+12} .
$$

By virtue of (3.1) and (3.7), $J_{T}(\varrho)$ satisfies for $\varrho \geqslant \varrho_{1}$ the estimate

$$
J_{T}\left(\varrho+J_{T}(\varrho)\right)<\theta J_{T}(\varrho)
$$

with the parameter $\theta=\left(\frac{C_{1}}{1+C_{1}}\right)^{\beta / m}$. Here, $C_{1}=C_{1}(n, N, \tilde{K})$ is the constant which appeared in (3.1). Lemma 3.2 entails that

$$
J_{T}(\varrho)=0
$$

for all

$$
\varrho>\varrho_{1}+\frac{J_{T}\left(\varrho_{1}\right)}{1-\theta}
$$

As a consequence,

$$
R(T) \leqslant \varrho_{1}+\frac{J_{T}\left(\varrho_{1}\right)}{1-\theta} .
$$

Inserting the definition of $J_{T}\left(\varrho_{1}\right)$, we find that

$$
R(T)-\varrho_{1} \leqslant C(n, N, \tilde{K})\left(R(T)-\varrho_{1}\right)^{\frac{2(n+2)}{(6+N) n+12}} T^{\frac{2}{(6+N) n+12}} \cdot I_{T}\left(\varrho_{1}\right)^{\frac{n}{(6+N) n+12}} .
$$

Rewriting (3.11) gives

$$
\left(R(T)-\varrho_{1}\right)^{\frac{(4+N) n+8}{(6+N) n+12}} \leqslant C(n, N, \tilde{K}) T^{\frac{2}{(6+N) n+12}} \cdot I_{T}\left(\varrho_{1}\right)^{\frac{n}{(6+N) n+12}},
$$

and (3.6) follows in an obvious way. 


\section{Estimates of $I_{T}(\varrho)$ by initial mass and proof of the theorem}

As the preliminary estimate (3.6) for the radius $R(T)$ of the solution's support at time $T>0$ still depends on the free parameter $\varrho_{1}$, let us look for estimates of $I_{T}(\varrho)$ in terms of $T$ and the initial mass. We will use the following auxiliary result.

LEMMA 4.1 Let $u$ be the solution of (1.1) considered before and assume that the parameters $\varrho, \delta$ satisfy $\varrho>R_{0}$ and $0<\delta<\varrho$. Then, there exists a positive constant $C=C(n, N)$ such that

$$
\begin{aligned}
\sup _{t \in(0, T)} \int_{\mathbb{R}^{N} \backslash B(\varrho+\delta)}|\nabla u(\cdot, t)|^{2} & +\int_{0}^{T} \int_{\mathbb{R}^{N} \backslash B(\varrho+\delta)}\left|\nabla u^{\frac{n+2}{6}}\right|^{6} \\
& \leqslant \frac{C}{\delta^{N(n+1)+6}}\left\|u_{0}\right\|_{L^{1}}^{n+2} \cdot T .
\end{aligned}
$$

Proof. Writing again $w:=u^{\frac{n+2}{6}}$ and using Lemma A.2 for values of $\varrho>R_{0}$ and $0<\delta<\varrho$ (note that $\varrho+\delta<2 \varrho$ !), we find that

$$
\begin{aligned}
&\left(\int_{A(\varrho, \varrho+\delta)} w^{6}\right)^{1 / 6} \leqslant C\left(\int_{A(\varrho, \varrho+\delta)}|\nabla w|^{6}\right)^{\frac{\Theta}{6}}\left(\int_{A(\varrho, \varrho+\delta)} w^{\frac{6}{n+2}}\right)^{\frac{n+2}{6}(1-\Theta)} \\
&+C \delta^{-N\left(\frac{n+2}{6}-\frac{1}{6}\right)}\left(\int_{A(\varrho, \varrho+\delta)} w^{\frac{6}{n+2}}\right)^{\frac{n+2}{6}}
\end{aligned}
$$

with $\Theta=\frac{N(n+1)}{N(n+1)+6}$. Hence,

$$
\begin{aligned}
\int_{A(\varrho, \varrho+\delta)} u^{n+2} \leqslant & C\left(\int_{A(\varrho, \varrho+\delta)}\left|\nabla u^{\frac{n+2}{6}}\right|^{6}\right)^{\frac{N(n+1)}{N(n+1)+6}}\left(\int_{A(\varrho, \varrho+\delta)} u\right)^{\frac{6(n+2)}{N(n+1)+6}} \\
& +\frac{C}{\delta^{N(n+1)}}\left(\int_{A(\varrho, \varrho+\delta)} u\right)^{n+2} .
\end{aligned}
$$

By conservation of mass, we infer that

$$
\begin{aligned}
\int_{0}^{T} \int_{A(\varrho, \varrho+\delta)} u^{n+2} \leqslant C & \left(\int_{0}^{T} \int_{A(\varrho, \varrho+\delta)}\left|\nabla u^{\frac{n+2}{6}}\right|^{6}\right)^{\frac{N(n+1)}{N(n+1)+6}}\left(\left\|u_{0}\right\|_{L^{1}\left(\mathbb{R}^{N}\right)}^{n+2} T\right)^{\frac{6}{N(n+1)+6}} \\
& +\frac{C}{\delta^{N(n+1)}}\left\|u_{0}\right\|_{L^{1}\left(\mathbb{R}^{N}\right)}^{n+2} \cdot T .
\end{aligned}
$$

Together with Young's inequality and (2.6) it follows that

$$
\begin{aligned}
\sup _{t \in(0, T)} \int_{\mathbb{R}^{N} \backslash B(\varrho+\delta)}|\nabla u(\cdot, t)|^{2}+ & d(n, N) \int_{0}^{T} \int_{\mathbb{R}^{N} \backslash B(\varrho+\delta)}\left|\nabla u^{\frac{n+2}{6}}\right|^{6} \\
\leqslant & \varepsilon \int_{0}^{T} \int_{A(\varrho, \varrho+\delta)}\left|\nabla u^{\frac{n+2}{6}}\right|^{6} \\
& +\left(C(n, N)+C(n, N)^{\frac{N(n+1)+6}{6}} C_{\varepsilon}\right) \frac{\left\|u_{0}\right\|_{L^{1}}^{n+2} \cdot T}{\delta^{N(n+1)+6}} .
\end{aligned}
$$


Obviously, this estimate also holds for $\varrho^{\prime}:=\varrho+\frac{\delta}{2}$ and $\delta^{\prime}:=\frac{\delta}{2}$. We rewrite it in the following way:

$$
\begin{aligned}
\sup _{t \in(0, T)} \int_{\mathbb{R}^{N} \backslash B(\varrho+\delta)}|\nabla u(\cdot, t)|^{2} & +d(n, N) \int_{0}^{T} \int_{\mathbb{R}^{N} \backslash B(\varrho+\delta)}\left|\nabla u^{\frac{n+2}{6}}\right|^{6} \\
& \leqslant \varepsilon \int_{0}^{T} \int_{\mathbb{R}^{N} \backslash B\left(\varrho+\frac{\delta}{2}\right)}\left|\nabla u^{\frac{n+2}{6}}\right|^{6}+\frac{C_{\varepsilon}\left\|u_{0}\right\|_{L^{1}}^{n+2} \cdot T}{\left(\frac{\delta}{2}\right)^{N(n+1)+6}} .
\end{aligned}
$$

Introducing

$$
G(\delta):=\delta^{N(n+1)+6} \sup _{t \in(0, T)} \int_{\mathbb{R}^{N} \backslash B(\varrho+\delta)}|\nabla u(\cdot, t)|^{2}
$$

and

$$
H(\delta):=\delta^{N(n+1)+6} \int_{0}^{T} \int_{\mathbb{R}^{N} \backslash B(\varrho+\delta)}\left|\nabla u^{\frac{n+2}{6}}\right|^{6}
$$

and choosing $\varepsilon:=d(n, N) 2^{-(N(n+1)+7)}$, we find using the iteration procedure presented in [20] that

$$
G(\delta)+d(n, N) H(\delta) \leqslant \frac{d(n, N)}{2^{j}} H\left(\frac{\delta}{2^{j}}\right)+C(\varepsilon, n, N) \sum_{i=0}^{j} \frac{\left\|u_{0}\right\|_{L^{1}}^{n+2} T}{2^{i}}
$$

for $j \in \mathbb{N}$, and in the limit $j \rightarrow \infty$ relation (4.1) is obtained.

This permits us to estimate $I_{T}(\varrho+\delta)$ in terms of the initial mass.

LEMMA 4.2 Let $u$ be the regular strong solution considered before. Then the following estimate holds for $T>0$ and parameters $\varrho, \delta$ satisfying $\varrho>R_{0}$ and $0<\delta<\varrho$ :

$$
I_{T}(\varrho+\delta) \leqslant C(n, N) \frac{\left\|u_{0}\right\|_{L^{1}}^{n+2} \cdot T}{\delta^{N(n+1)}} .
$$

Proof. The homogeneous Gagliardo-Nirenberg inequality on exterior domains (see e.g. [19: Theorem 7.4.1]) combined with (4.1) gives

$$
\begin{aligned}
\int_{0}^{T} \int_{\mathbb{R}^{N} \backslash B(\varrho+\delta)} u^{n+2} & \leqslant C(n, N)\left(\left\|u_{0}\right\|_{L^{1}}\right)^{\frac{6(n+2)}{N(n+1)+6}} T^{\frac{6}{N(n+1)+6}}\left(\int_{0}^{T} \int_{\mathbb{R}^{N} \backslash B(\varrho+\delta)}\left|\nabla u^{\frac{n+2}{6}}\right|^{6}\right)^{\frac{N(n+1)}{N(n+1)+6}} \\
& \leqslant C(n, N)\left(\left\|u_{0}\right\|_{L^{1}}\right)^{\frac{6(n+2)}{N(n+1)+6}} T^{\frac{6}{N(n+1)+6}}\left(\frac{\left\|u_{0}\right\|_{L^{1}}^{n+2} \cdot T}{\delta^{N(n+1)+6}}\right)^{\frac{N(n+1)}{N(n+1)+6}} \\
& =C(n, N)\left\|u_{0}\right\|_{L^{1}}^{n+2} \cdot T \delta^{-N(n+1)} .
\end{aligned}
$$

Now we have collected all the estimates necessary to prove the main result.

Proof of Theorem 1.3. Let us replace $\varrho+\delta$ by $\varrho^{\prime}$ in (4.3) and let us choose $\delta^{\prime}=\varrho^{\prime}-R_{0}$. Then (4.3) becomes

$$
I_{T}\left(\varrho^{\prime}\right) \leqslant \frac{C(n, N)\left\|u_{0}\right\|_{L^{1}}^{n+2} \cdot T}{\left(\varrho^{\prime}-R_{0}\right)^{N(n+1)}}
$$


for all $\varrho^{\prime}>R_{0}$. Combined with (3.6), we obtain the following estimate for $R(T)$, provided

$$
\begin{gathered}
\varrho>\max \left\{R_{0}, \frac{R(T)}{\tilde{K}}\right\} . \\
R(T) \leqslant \varrho+C(n, N, \tilde{K}) T^{\frac{2}{(4+N) n+8}}\left(\frac{C(n, N, \tilde{K})\left\|u_{0}\right\|_{L^{1}}^{n+2} \cdot T}{\left(\varrho-R_{0}\right)^{N(n+1)}}\right)^{\frac{n}{(4+N) n+8}} \\
=\varrho+\frac{C(n, N, \tilde{K}) T^{\frac{22+n}{(4+N) n+8}}\left(\left\|u_{0}\right\|_{L^{1}}\right)^{\frac{n(n+2)}{(4+N) n+8}}}{\left(\varrho-R_{0}\right)^{\frac{n N(n+1)}{(4+N) n+8}}} .
\end{gathered}
$$

Let us rewrite (4.5) in the form

$$
R(T) \leqslant \varrho+\frac{S(T)}{\left(\varrho-R_{0}\right)^{\alpha}} \text { for } \varrho>\max \left\{\frac{R(T)}{\tilde{K}}, R_{0}\right\}
$$

with

$$
S(T):=C(n, N, \tilde{K}) \cdot T^{\frac{2+n}{(4+N) n+8}\left\|u_{0}\right\|_{L^{1}}^{\frac{n(n+2)}{(4+N) n+8}}}
$$

and

$$
\alpha:=\frac{n N(n+1)}{(4+N) n+8} .
$$

Our strategy is to minimize the right-hand side of (4.6) for values of $\varrho$ contained in the interval $\left(\max \left\{\frac{R(T)}{\tilde{K}}, R_{0}\right\}, \infty\right)$.

For further reference, let us choose $\tilde{K}$ once and for all times such large that

$$
(1-\tilde{K}) \alpha^{\frac{1}{\alpha+1}}+\alpha^{-\frac{\alpha}{\alpha+1}}<0 .
$$

This can easily be accomplished as $\alpha$ does not depend on $\tilde{K}$. Note that the function

$$
f_{T}(\varrho):=\varrho+\frac{S(T)}{\left(\varrho-R_{0}\right)^{\alpha}}
$$

has a global minimum $\varrho_{*}(T)$ on the interval $\left(R_{0}, \infty\right)$ which is attained in

$$
\varrho_{*}(T)=R_{0}+\alpha^{\frac{1}{\alpha+1}} \cdot S(T)^{\frac{1}{\alpha+1}} .
$$

As a consequence, the minimum value of $f_{T}$ on $\left(R_{0}, \infty\right)$ is given by

$$
\min _{\varrho \in\left(R_{0}, \infty\right)} f_{T}(\varrho)=R_{0}+\left(\alpha^{\frac{1}{\alpha+1}}+\alpha^{-\frac{\alpha}{\alpha+1}}\right) \cdot S(T)^{\frac{1}{\alpha+1}} .
$$

As $S(T)$ is increasing in $T$ with $S(0)=0$, for small values of $T$ the inequality

$$
\varrho_{*}(T)>\max \left\{\frac{R(T)}{\tilde{K}}, R_{0}\right\}
$$


is satisfied. Let us prove that (4.10) is in fact true for all $T>0$.

Assuming the contrary, there would exist a positive number $T^{*}$ such that

$$
\begin{aligned}
\varrho_{*}(T) & >\max \left\{\frac{R(T)}{\tilde{K}}, R_{0}\right\}, \\
R(T) & \leqslant R_{0}+\left(\alpha^{\frac{1}{\alpha+1}}+\alpha^{-\frac{\alpha}{\alpha+1}}\right) \cdot S(T)^{\frac{1}{\alpha+1}}
\end{aligned}
$$

for all $0<T<T^{*}$, but

$$
\varrho_{*}\left(T^{*}\right) \leqslant \max \left\{\frac{R\left(T^{*}\right)}{\tilde{K}}, R_{0}\right\}=\frac{R\left(T^{*}\right)}{\tilde{K}} .
$$

By continuity,

$$
R\left(T^{*}\right) \leqslant R_{0}+\left(\alpha^{\frac{1}{\alpha+1}}+\alpha^{-\frac{\alpha}{\alpha+1}}\right) \cdot S\left(T^{*}\right)^{\frac{1}{\alpha+1}}
$$

and

$$
R\left(T^{*}\right) \geqslant \tilde{K} \cdot \varrho_{*}\left(T^{*}\right)=\tilde{K} \cdot R_{0}+\tilde{K} \alpha^{\frac{1}{\alpha+1}} S\left(T^{*}\right)^{\frac{1}{\alpha+1}},
$$

which gives

$$
(\tilde{K}-1) R_{0} \leqslant\left((1-\tilde{K}) \alpha^{\frac{1}{\alpha+1}}+\alpha^{-\frac{\alpha}{\alpha+1}}\right) \cdot S\left(T^{*}\right)^{\frac{1}{\alpha+1}} .
$$

The latter inequality contradicts (4.7) and therefore (4.10) is true for all $T>0$.

Especially in our case, we compute using the definition of $\alpha$ and $S(T)$ :

$$
\begin{aligned}
R(T) & \leqslant R_{0}+C(n, N, \tilde{K}) T^{\frac{2+n}{N n^{2}+(4+2 N) n+8}} \cdot\left(\left\|u_{0}\right\|_{L^{1}}\right)^{\frac{n(n+2)}{N n^{2}+(2 N+4) n+8}} \\
& =R_{0}+C(n, N, \tilde{K}) \cdot\left(\left\|u_{0}\right\|_{L^{1}}\right)^{\frac{n}{4+n N}} \cdot T^{\frac{1}{4+n N}} .
\end{aligned}
$$

This proves the theorem.

\section{Acknowledgement}

This work has partially been supported by grant Gr1693/1-1 of the priority programme Benetzung und Strukturbildung an Grenzfächen of Deutsche Forschungsgemeinschaft.

\section{REFERENCES}

1. Andreucci, D. \& Tedeev, A. F. Finite speed of propagation for the thin-film equation and other higher-order parabolic equations with general nonlinearity. Interfaces and Free Boundaries 3, (2001) 233-264.

2. Beretta, E., Bertsch, M., \& Dal Passo, R. Nonnegative solutions of a fourth order nonlinear degenerate parabolic equation. Arch. Ration. Mech. Anal. 129, (1995) 175-200.

3. BERNIS, F. Finite speed of propagation and continuity of the interface for thin viscous flows. $A d v$. Differential Equations 1, (1996) 337-368.

4. BERnis, F. Finite speed of propagation for thin viscous flows when $2 \leqslant n<3$. C.R. Acad. Sci. Paris; Sér. I Math. 322, (1996). 
5. BERNIS, F. Integral inequalities with applications to nonlinear degenerate parabolic equations. In: Angell, T. S., Cook, L. P., Kleinman, R. E. \& Olmstead, W. E. (eds), Nonlinear Boundary Value Problems. SIAM, Philadelphia, PA (1996).

6. Bernis, F. \& Friedman, A. Higher order nonlinear degenerate parabolic equations. J. Differential Equations 83, (1990) 179-206.

7. Bernis, F., Peletier, L. A., \& Williams, S. M. Source-type solutions of a fourth order nonlinear degenerate parabolic equation. Nonlinear Anal. 18, (1992) 217-234.

8. Bertozzi, A. L. \& Pugh, M. The lubrication approximation for thin viscous films: regularity and long time behaviour of weak solutions. Comm. Pure Appl. Math. 49, (1996) 85-123.

9. Bertsch, M., Dal Passo, R., Garcke, H., \& Grün, G. The thin viscous flow equation in higher space dimensions. Adv. Differential Equations 3, (1998) 417-440.

10. Dal Passo, R., Garcke, H., \& GRÜN, G. On a fourth order degenerate parabolic equation: global entropy estimates and qualitative behaviour of solutions. SIAM J. Math. Anal. 29, (1998) 321-342.

11. Dal Passo, R., Giacomelli, L., \& GRÜN, G. A waiting time phenomenon for thin film equations. Ann. Scuola Norm. Sup. Pisa XXX, (2001) 437-463.

12. Dal Passo, R., Giacomelli, L., \& Shishkov, A. The thin film equation with nonlinear diffusion. Comm. PDE 26, (2001) 1509-1557.

13. DE Gennes, P. G. Wetting: statistics and dynamics. Rev. Modern Phys. 57, (1985) 827-863.

14. Dussan, E. B. \& DAVIS, S. On the motion of a fluid-fluid interface along a solid surface. J. Fluid Mech. 65, (1974) 71-95.

15. FERREIRA, R. \& Bernis, F. Source-type solutions to thin-film equations in higher space dimensions. European J. Appl. Math. 8, (1997) 507-524.

16. Giacomelli, L. \& Otto, F. Droplet spreading: intermediate scaling law by pde methods. Comm. Pure Appl. Math. 55, (2002) 217-254.

17. GRÜN, G. Droplet spreading under weak slippage: a basic result on finite speed of propagation. submitted for publication.

18. GRÜN, G. On Bernis' interpolation inequalities in multiple space dimensions. Z. Anal. Anwendungen 20, (2001) 987-998.

19. GRÜN, G. On free boundary problems arising in thin film flow, Habilitation thesis, University of Bonn, (2001).

20. Hulshof, J. \& Shishrov, A. The thin film equation with $2 \leqslant n<3$ : finite speed of propagation in terms of the $L^{1}$-norm. Adv. Diff. Equations 3, (1998) 625-642.

21. Murthy, M. K. V. \& Stampacchia, G. Boundary value problems for some degenerate elliptic operators. Ann. di Matematica 80, (1968) 1-122.

22. Oron, A., Davis, S. H., \& BanKoff, S. G. Long-scale evolution of thin liquid films. Reviews of Modern Physics 69, (1997) 932-977.

\section{Appendix}

For the reader's convenience, let us prove the following version of Poincaré's inequality valid on annuli in $\mathbb{R}^{N}$.

Lemma A.1 Let $A=B\left(R_{1}\right) \backslash \overline{B\left(R_{0}\right)}, R_{1}>R_{0}>0$, be an annulus in $\mathbb{R}^{N}$ and assume

$$
R_{1}<K R_{0}
$$

for a real number $K>1$. Suppose in addition that $1<p<\infty$. Then a positive constant $C=$ $C(N, K)$ exists such that the following estimate is true for all $u \in W^{1, p}(A)$ which vanish on 
$\partial B\left(R_{1}\right)$ in the sense of traces.

$$
\int_{A}|u(x)|^{p} \mathrm{~d} x \leqslant C\left(R_{1}-R_{0}\right)^{p} \int_{A}|\nabla u(x)|^{p} \mathrm{~d} x .
$$

Proof. It will be sufficient to prove the result for smooth functions $u$ satisfying $\left.u\right|_{\partial B\left(R_{1}\right)} \equiv 0$. Denoting the surface element on $S^{N-1}$ by $\mathrm{d} S^{N-1}$, abbreviating the angular coordinates by $\Omega$ and writing $\tilde{u}$ for the function in polar coordinates corresponding to $u$, we find for $r \in\left[R_{0}, R_{1}\right]$ :

$$
\tilde{u}(r, \Omega)=-\int_{r}^{R_{1}} \tilde{u}_{r}(s, \Omega) \mathrm{d} s .
$$

Hence,

$$
\begin{aligned}
|\tilde{u}(r, \Omega)|^{p} & \leqslant\left|r-R_{1}\right|^{p-1} \int_{r}^{R_{1}}\left|\tilde{u}_{r}(s, \Omega)\right|^{p} \mathrm{~d} s \\
& \leqslant\left(R_{1}-R_{0}\right)^{p-1} \int_{R_{0}}^{R_{1}}\left|\tilde{u}_{r}(s, \Omega)\right|^{p} \mathrm{~d} s .
\end{aligned}
$$

As a consequence,

$$
\begin{aligned}
\int_{A}|u(x)|^{p} \mathrm{~d} x & =\int_{R_{0}}^{R_{1}} \int_{S^{N-1}}|\tilde{u}(s, \Omega)|^{p} s^{N-1} \mathrm{~d} \Omega \mathrm{d} s \\
& \leqslant C(N)\left(R_{1}-R_{0}\right)^{N+p-1} \int_{S^{N-1}} \int_{R_{0}}^{R_{1}}\left|\tilde{u}_{r}(s, \Omega)\right|^{p} \mathrm{~d} s \mathrm{~d} \Omega \\
& \leqslant C(N)(K-1)^{N-1}\left(R_{1}-R_{0}\right)^{p} \int_{S^{N-1}} \int_{R_{0}}^{R_{1}}\left|\tilde{u}_{r}(s, \Omega)\right|^{p} s^{N-1} \mathrm{~d} s \mathrm{~d} \Omega \\
& \leqslant C(N, K)\left(R_{1}-R_{0}\right)^{p} \int_{A}|\nabla u|^{p} \mathrm{~d} x
\end{aligned}
$$

where we used (A.1) in the second step of this chain of estimates.

In addition, we cite the following version of Gagliardo-Nirenberg's inequality which can be found in [12].

LemMA A.2 Let $1 \leqslant r \leqslant \infty, p>0, q \in(0, p), m \in \mathbb{N}_{+}$such that

$$
\frac{1}{r}-\frac{m}{N}<\frac{1}{p} \text {. }
$$

If $\Omega \subset \mathbb{R}^{N}$ is bounded with piecewise smooth boundary, then positive constants $c_{1}$ and $c_{2}$ depending only on $\Omega, r, p, m$ and $q$ exist such that for any $u \in L^{q}(\Omega)$ satisfying $D^{m} u \in L^{r}(\Omega)$, the following inequality holds:

$$
\|u\|_{p} \leqslant c_{1}\left\|D^{m} u\right\|_{r}^{a}\|u\|_{q}^{1-a}+c_{2}\|u\|_{q}
$$

where $a=\frac{\frac{1}{q}-\frac{1}{p}}{\frac{1}{q}+\frac{m}{N}-\frac{1}{r}}$.

In addition, a positive constant $c=c(r, p, m, q)$ exists such that 
(i) if $\Omega=B_{R}(0)$, then (A.3) holds with

$$
c_{1}=c \text { and } c_{2}=c \cdot R^{-N\left(\frac{1}{q}-\frac{1}{p}\right)},
$$

(ii) if $\Omega=\mathbb{R}^{N}$, then the result holds with $c_{1}=c$ and $c_{2}=0$,

(iii) if $\Omega=B_{R_{2}}(0) \backslash B_{R_{1}}(0)$ with $0<R_{1}<R_{2}$ where we require $3 R_{1}>R_{2}$ if $N>1$, then (A.3) holds with

$$
c_{1}=c, c_{2}=c\left(R_{2}-R_{1}\right)^{-N\left(\frac{1}{q}-\frac{1}{p}\right)} .
$$

Finally, if $\Omega$ is an infinite cone, i.e. for given points $x_{0}, y_{0} \in \mathbb{R}^{N}, x_{0} \notin B_{1}\left(y_{0}\right)$ a set

$$
C_{x_{0}, y_{0}}:=\left\{z \in \mathbb{R}^{N} \mid z=x_{0}+\lambda\left(y-x_{0}\right), \quad y \in B_{1}\left(y_{0}\right), \quad \lambda>0\right\},
$$

then (A.3) holds with constants $c_{1}=c\left(\left\|x_{0}-y_{0}\right\|, r, p, m, q\right)$ and $c_{2}=0$. 